\title{
Plastía diafragmática y pleurodesis química por videotoracoscopia: una alternativa como tratamiento quirúrgico en paciente con hidrotórax hepático
}

\section{Chemical diaphragmoplasty and pleurodesis by videotoracoscopy as a surgical treatment in a patient with liver hydrothorax}

\author{
José Antonio Rodríguez-Blanco, ${ }^{*}$ Yulieth Acevedo-Pérez, \\ Jonathan Rodríguez-Blanco, ${ }^{*}$ Carlos Remolina-Medina*
}

*Barrio Los Corales-Cartagena/Colombia.

RESUMEN. El hidrotórax hepático es una patología de difícil diagnóstico de la que muy poco se sabe por su baja incidencia. Presentamos un caso clínico de un paciente con un hidrotórax hepático, donde se consideró como alternativa el tratamiento quirúrgico con diafragmoplastía más pleurodesis química por videotoracoscopia, debido a su poca adherencia con el tratamiento médico, presentando recurrencias del hidrotórax y hospitalizaciones frecuentes. Esta cirugía se presenta como una alternativa con buenos resultados durante un importante período de tiempo, mejorando la calidad de vida del paciente.

Palabras clave: Hidrotórax hepático, plastía diafragmática, videotoracoscopia.

\section{INTRODUCCIÓN}

El hidrotórax hepático $(\mathrm{HH})$ se debe a una regulación anómala del volumen del líquido extracelular, que en muchos casos resulta en acumulación de éste en la cavidad abdominal, generando el desarrollo de ascitis, formación de edema en las extremidades inferiores, y posteriormente conlleva a la acumulación del líquido en el espacio pleural, el cual se presenta en el $5-10 \%$ de los casos.

\section{Correspondencia:}

\section{Dr. José Antonio Rodríguez Blanco}

Barrio Amberes-Cartagena/Colombia.

Correo electrónico: jose7080@hotmail.com

Recibido: 01-VIII-2020; aceptado: 07-IX-2020.

Citar como: Rodríguez-Blanco JA, Acevedo-Pérez Y, Rodríguez-Blanco J, Remolina-Medina C. Plastía diafragmática y pleurodesis química por videotoracoscopia: una alternativa como tratamiento quirúrgico en paciente con hidrotórax hepático. Neumol Cir Torax. 2021; 80 (1):46-50. https://dx.doi. org/10.35366/99454
ABSTRACT. Hepatic hydrothorax is a difficult diagnosis pathology, of which very little is known due to its low incidence. Next, we will present a clinical case of a patient with a hepatic hydrothorax, where surgical treatment with diaphragmoplasty plus chemical pleurodesis by videothoracoscopy was considered as an alternative, due to its low adherence to medical treatment, presenting recurrences of the hydrothorax and frequent hospitalizations. This surgery is presented as an alternative with good results for an important period of time, improving the quality of life of the patient

Keywords: Hydrothorax hepatic, diaphragmoplasty, videothoracoscopy.

Es una patología con una gran mortalidad y una mediana de supervivencia desde su detección de 9.1 meses. ${ }^{2}$ La incidencia de esta entidad varía de acuerdo con el método usado para el diagnóstico, se identifica aproximadamente en un 4 a $6 \%$ de los pacientes con cirrosis hepática a quienes se les realiza una radiografía de tórax. ${ }^{3}$

La hipoalbuminemia, el incremento en el flujo, la presión elevada en el conducto torácico y las venas ácigos son eventos comunes en los pacientes cirróticos con hipertensión portal, podrían explicar en parte el desarrollo de hidrotórax. Sin embargo, la causa más probable es la transferencia de grandes volúmenes de líquido, desde el abdomen al espacio pleural por medio de un gradiente de presión a través de frenestraciones congénitas o adquiridas en el diafragma. ${ }^{4}$

Hay un fino balance entre la formación y la absorción del líquido pleural, cuando éste se altera se desarrolla el derrame pleural. La presión negativa intratorácica generada durante la inspiración, asociada a la presión positiva intraabdominal, favorece el paso de líquido desde la cavidad abdominal hasta el espacio pleural. Algunos casos pueden tener ascitis leve o incluso no detectable. Cuando se excede 
la capacidad de absorción del espacio pleural se desarrolla el hidrotórax.

El HH es más frecuente en el hemitórax derecho en un $79.5 \%$, aunque puede ser izquierdo en un $17.5 \%$ y bilateral en el $3 \%$.

\section{PRESENTACIÓN DEL CASO}

Se trata de un hombre de 54 años, con antecedente de cirrosis hepática diagnosticada hace aproximadamente siete años, que ingresó a la Unidad de Cuidados Intensivos por encefalopatía hepática tipo C episódica recurrente, grado III según la clasificación West Haven, ascitis y derrame pleural derecho masivo (Figura 1). Dentro de los antecedentes, presentaba una cirrosis hepática de origen alcohólica, recibiendo tratamiento irregular con disacáridos no absorbibles, espironolactona $25 \mathrm{mg} /$ día y sin cumplir con las recomendaciones nutricionales, además de hipertensión arterial y diabetes mellitus tipo 2 controlada según los familiares, recibiendo losartán 50 mg cada 12 horas y metformina 850 mg cada 12 horas. Los síntomas iniciaron una semana antes de la hospitalización en la Unidad de Cuidados Intensivos ( $\mathrm{UCl}$ ) consistentes en ansiedad, confusión mental, alteraciones en el lenguaje y estupor, además

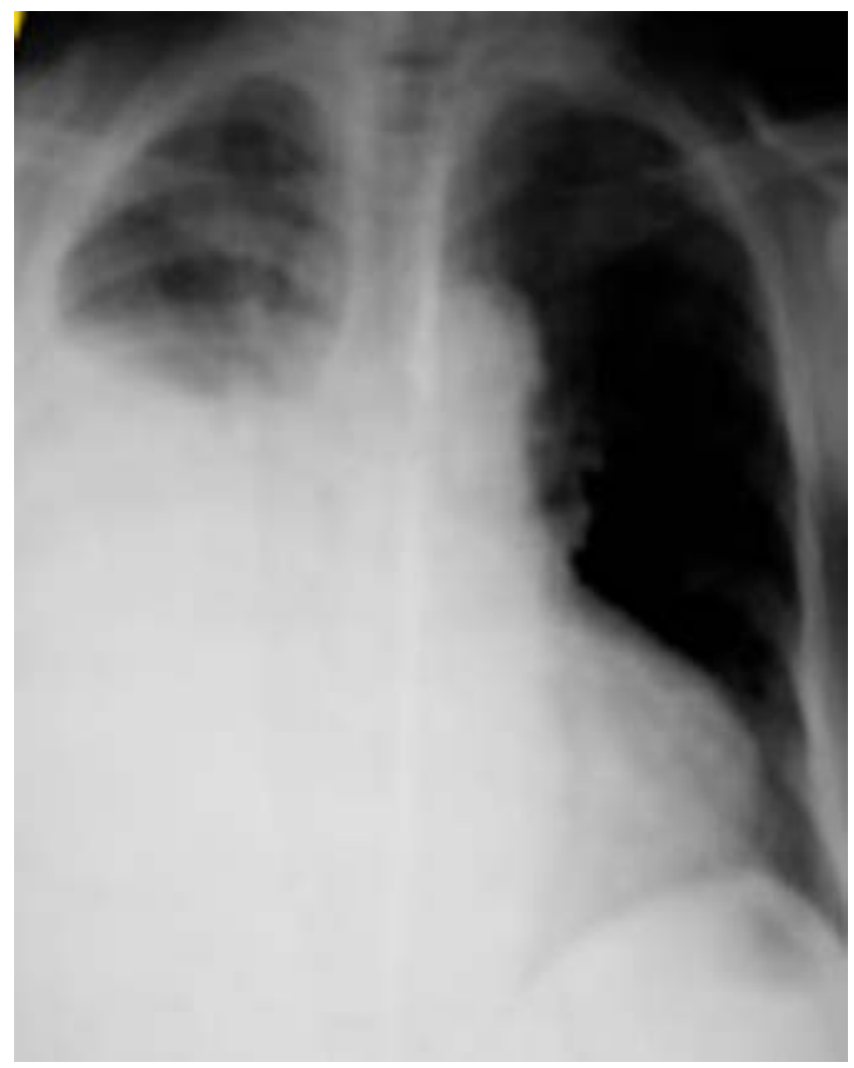

Figura 1: Derrame pleural derecho masivo. de disnea en reposo, constipación y dolor torácico de tipo opresivo; al ingreso, los gases arteriales iniciales eran compatibles con hipoxemia moderada y acidosis metabólica. En principio fue tratado por el Servicio de Medicina Crítica con vigilancia hemodinámica y ventilatoria, fue iniciado con oxígeno terapia de alto flujo con máscara de ventury al 50\%, diuréticos con furosemida 20 mg IV cada 12 horas, reposición de albumina, disacáridos no absorbibles $20 \mathrm{~g}$ cada ocho horas, restricción de sodio y líquidos.

Fue solicitada valoración por cirugía general, donde realizaron paracentesis terapéutica y toracostomía cerrada, con mejora notable de los síntomas respiratorios. El líquido pleural fue compatible con un transudado, el Gram y el cultivo para gérmenes comunes del líquido pleural fueron negativos, el débito diario por el tubo de tórax era entre 1,000-1,500 mL de líquido pleural cetrino durante cinco días a pesar del tratamiento médico, por lo que es valorado por cirugía de tórax.

Al examen físico tenía un tubo de tórax derecho número $28 \mathrm{Fr}$, a la auscultación se identifica ausencia del murmullo vesicular en base pulmonar derecha, abdomen un poco distendido. Teniendo en cuenta todo el contexto del paciente, los antecedentes de cirrosis hepática, ascitis (hipertensión arterial y diabetes mellitus controlada), estudios imagenológicos (ecocardiograma transtorácico normal), estudio del líquido pleural compatible con un transudado y la poca respuesta al tratamiento médico, se realiza diagnóstico de $\mathrm{HH}$ refractario.

Se consideró tratamiento quirúrgico con plastía diafragmática y pleurodesis química por videotoracoscopia debido al fracaso y resistencia al tratamiento médico para la cirrosis, utilizando la siguiente técnica:

1. Anestesia general-inducción propofol $1.5 \mathrm{mg} / \mathrm{kg}$; lidocaína simple $1 \mathrm{mg} / \mathrm{kg}$; cisatracurio $0.1 \mathrm{mg} / \mathrm{kg}$ - mantenimiento con remifentanil $0.15-0.25 \mu \mathrm{g} / \mathrm{kg} / \mathrm{min}$; sevoflorane 0.5-0.6 MAC. Asepsia y antisepsia con clorexidina espuma y solución, campos quirúrgicos estériles.

2. Intubación selectiva con tubo de doble luz izquierdo número $37 \mathrm{Fr}$, el paciente se posicionó en decúbito lateral izquierdo.

3. Se realizaron puertos de videotoracoscopia: el lente fue colocado en el cuarto espacio intercostal con línea axilar anterior derecha y puerto de trabajo de $3 \mathrm{~cm}$ en séptimo espacio intercostal con línea axilar posterior derecha.

4. Se drenó el derrame pleural. Se identificó el diafragma sin presencia de fenestraciones visibles.

5. Se realizó plastía diafragmática con sutura no absorbible (polipropileno 1-0) en dos planos y colocación de malla de polipropileno no absorbible (Figura $2 A$ y B).

6. Fueron realizadas pleurodesis químicas con talco (5 g) con aspersor, hasta cubrir toda la cavidad pleural. 
Figura 2:

A) Plastía diafragmática en dos planos. B) Colocación de malla de polipropileno no absorbible.
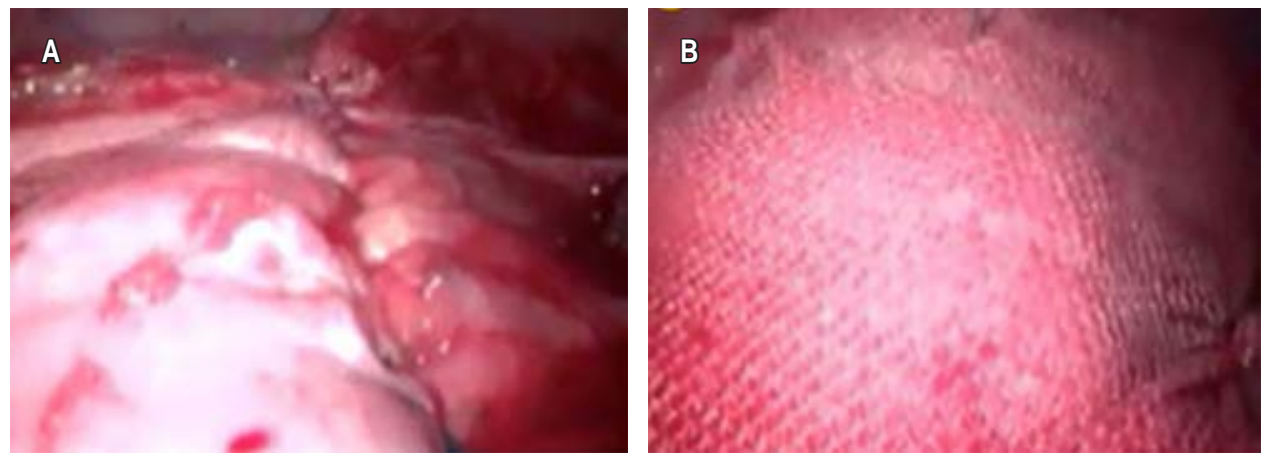

7. Se revisó cavidad pleural, se colocó tubo de tórax número $28 \mathrm{Fr}$. Se conectó a un pleurovac, cierre por planos muscular (poliglactina 1-0), piel (poliglecaprone 3-0).

El paciente presentó un posoperatorio satisfactorio con dos días de estancia en $\mathrm{UCl}$ y tres días en sala general. Al quinto día presentó disminución del drenaje por el tubo de tórax, el cual fue menos de $100 \mathrm{~mL}$, se retira el tubo y se da alta hospitalaria.

A los quince días del egreso, asiste a la consulta en buenas condiciones generales, sin sintomatología respiratoria, con persistencia de la ascitis, deambulando por sus propios medios, y radiografía control sin evidencia de hidrotórax (Figura 3). A los seis meses del seguimiento fallece por encefalopatía e insuficiencia hepática severa, sin recidiva del hidrotórax.

\section{DISCUSIÓN}

El HH hace referencia a la presencia de líquido pleural normalmente mayor a $500 \mathrm{~mL}$ con características de transudado, en un paciente con cirrosis hepática sin otras causas que expliquen la presencia de un derrame pleural (por ejemplo, ausencia de enfermedad cardíaca, pulmonar, o pleural), ${ }^{5,6}$ ocurre en aproximadamente 5 a 10\% de los pacientes con cirrosis.

Los mecanismos exactos que se encuentran involucrados en el desarrollo de esta patología aún se desconocen por completo; sin embargo, se puede presentar como resultado del paso del líquido ascítico de la cavidad peritoneal hacia la cavidad pleural a través de pequeños estomas diafragmáticos. Estos estomas pueden ser menores a $1 \mathrm{~cm}$ (pueden ser microscópicos) y por lo general se encuentran en la parte tendinosa del diafragma.,8

El HH se hace evidente cuando se excede la capacidad de absorción del líquido pleural en el espacio pleural. La presión intratorácica negativa generada durante la inspiración promueve el paso de líquido de la cavidad abdominal al espacio pleural. Esto podría explicar por qué algunos pacientes con $\mathrm{HH}$ no tienen ascitis a simple vista. ${ }^{9,10}$ Los defectos diafragmáticos se encuentran más a menudo en el hemidiafragma derecho, quizá debido al hecho de que el hemidiafragma izquierdo es más grueso y muscular. El HH se desarrolla en el lado derecho en aproximadamente un $73-85 \%$, mientras que en el lado izquierdo sólo en un 13-17\% y de forma bilateral en aproximadamente $2-10 \% \cdot{ }^{11-14}$

Las manifestaciones clínicas más comunes en pacientes con $\mathrm{HH}$ incluyen disnea, tos no productiva, dolor torácico tipo pleurítico y fatiga debido a la hipoxemia. ${ }^{15}$ Los casos a menudo tienen signos de ascitis, así como otros hallazgos asociados con cirrosis (eritema palmar). Las presentaciones menos comunes incluyen un hidrotórax a tensión que se asocia a disnea grave, hipotensión o a empiema bacteriano espontáneo, lo que está descrito en un 13 a 16\% de los casos. ${ }^{16}$

El diagnóstico de $\mathrm{HH}$ incluye la documentación de un derrame pleural de tipo transudado y la exclusión de otras posibles causas para el derrame pleural. El estudio inicial puede ser una radiografía de tórax, donde se va a documentar el derrame pleural. El HH es más frecuente del lado derecho, aunque puede ser bilateral. La toracentesis es importante para confirmar el tipo de derrame, así como imágenes adicionales como una tomografía computarizada de tórax y un ecocardiograma, lo que nos puede ayudar a excluir infección o causas alternativas de derrame pleural. Hasta un 20\% de los casos tendrá evidencia de infección u otra causa de derrame pleural que no esté relacionada con $\mathrm{HH}^{17}$

El tratamiento es similar al de la ascitis, los pacientes que están gravemente sintomáticos deben someterse a una toracentesis terapéutica, seguida de una dieta con restricción de sodio. ${ }^{18}$

El uso de toracostomía cerrada no está indicado en el tratamiento del $\mathrm{HH}$ porque puede dar lugar a la pérdida de proteínas y electrólitos de forma masiva, se asocia con infección, insuficiencia renal y hemorragia; aunque puede ser necesario para los pacientes con empiema pleural asociado o en aquéllos que van a ser sometidos a pleurodesis. ${ }^{19}$ 
Otra alternativa es la pleurodesis química, que se utiliza para tratar pacientes con derrames pleurales malignos, recurrentes y sintomáticos; sin embargo, hay poca información disponible para guiar al clínico en el manejo de los casos con derrame pleural no maligno recurrente. La experiencia sugiere que el $\mathrm{HH}$ es difícil de tratar con este método debido a la rápida migración de líquido del abdomen al espacio pleural. A menudo no es posible mantener las dos superficies pleurales unidas el tiempo suficiente para que el proceso inflamatorio produzca su adherencia definitiva. ${ }^{20}$

En los pacientes con $\mathrm{HH}$ persistente, a pesar de una dieta baja en sodio y tratamiento con diuréticos, o que desarrollen complicaciones relacionadas con diuréticos, se considera que tienen hidrotórax refractario al tratamiento. En estos casos, las opciones de manejo incluyen toracentesis repetidas, colocación de derivaciones portosistémicas (TIPS), pleurodesis química por el tubo de tórax, reparación quirúrgica de defectos en el diafragma y el trasplante de hígado. Los tratamientos alternativos deben ser considerados ya que las toracentesis repetidas aumentan el riesgo de complicaciones. ${ }^{21}$

En nuestro caso clínico cabe resaltar que el paciente tenía poca adherencia al tratamiento médico debido al entorno social en el que se encontraba, por lo que se descartó en él otros tipos de tratamientos quirúrgicos, los cuales se utilizan como primera opción en casos con esta patología, como el trasplante hepático o las TIPS.

Para resolver los problemas descritos anteriormente con la pleurodesis química, se decidió el uso de una técnica combinada, la plastía diafragmática con pleurodesis química por videotoracoscopia. Al realizar la técnica descrita, se cumple el primer objetivo de la cirugía, que es impedir el paso del líquido ascítico de la cavidad peritoneal al espacio pleural el suficiente tiempo para que la pleurodesis haga su efecto y se produzca la adherencia pleural, garantizando el éxito del tratamiento.

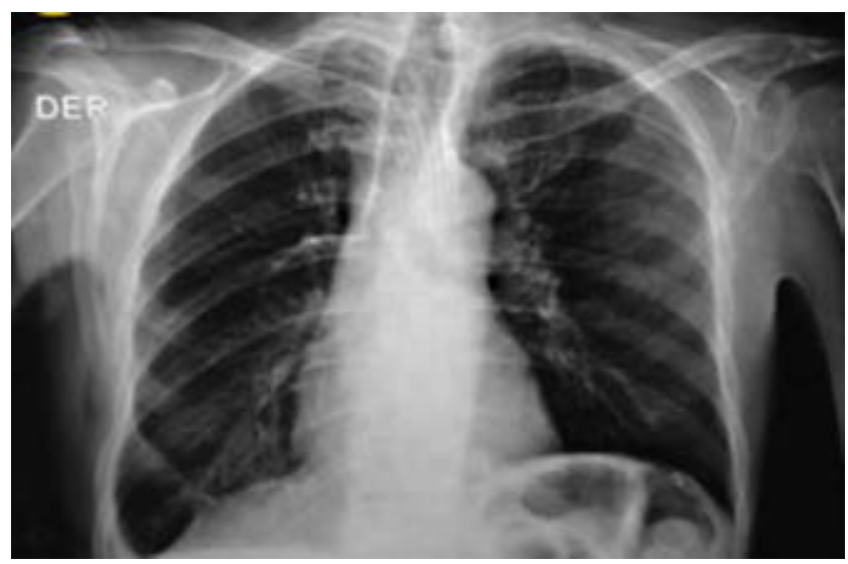

Figura 3: Rx de tórax control del posoperatorio.

\section{CONCLUSIONES}

El manejo del $\mathrm{HH}$ es difícil cuando es refractario al tratamiento médico, la diafragmoplastía combinada con pleurodesis química por videotoracoscopia es una excelente alternativa en pacientes que no tienen indicación de trasplante hepático o derivaciones portosistémicas, con muy buenos resultados, disminuyendo la frecuencia de hospitalizaciones y mejorando la calidad de vida.

\section{REFERENCIAS}

1. Porcel JM, Light RW. Pleural effusions. Dis Mon. 2013;59(2):29-57. https://doi.org/10.1016/j.disamonth.2012.11.002

2. Porcel JM, Mas E, Reñé JM, Bielsa S. Hidrotórax de causa hepática: análisis de 77 pacientes. Med Clin. 2013;141(11):484-486. https://doi. org/10.1016/j.medcli.2013.06.017.

3. Jiang SJ, Zhang S, Su LL, Liu Y. Diagnostic and therapeutic value of thoracoscopy for pleural effusions: experience from 628 consecutive cases in China. Chin Med J (Engl). 2009;122(10):1227-1230.

4. Cardenas A, Kelleher T, Chopra S. Review article: hepatic hydrothorax. Aliment Pharmacol Ther. 2004;20(3):271-279. https:// doi.org/10.1111/j.1365-2036.2004.02081.x.

5. Kinasewitz GT, Keddissi Jl. Hepatic hydrothorax. Curr Opin Pulm Med. 2003;9(4):261-265. https://doi.org/10.1097/00063198-20030700000003.

6. Badillo R, Rockey DC. Hepatic hydrothorax: clinical features, management, and outcomes in 77 patients and review of the literature. Medicine (Baltimore). 2014;93(3):135-142. https://doi.org/10.1097/ md.0000000000000025.

7. Huang PM, Chang YL, Yang CY, Lee YC. The morphology of diaphragmatic defects in hepatic hydrothorax: thoracoscopic finding. J Thorac Cardiovasc Surg. 2005;130(1):141-145. https://doi. org/10.1016/j.jtcvs.2004.08.051.

8. Lieberman FL, Hidemura R, Peters RL, Reynolds TB. Pathogenesis and treatment of hydrothorax complicating cirrhosis with ascites. Ann Intern Med. 1966;64(2):341-351. https://doi.org/10.7326/0003-481964-2-341.

9. Emerson PA, Davies JH. Hydrothorax complicating ascites. Lancet. 1955;268(6862):487-488. https://doi.org/10.1016/s01406736(55)90269-5.

10. Mouroux J, Perrin C, Venissac N, Blaive B, Richelme H. Management of pleural effusion of cirrhotic origin. Chest. 1996;109(4):1093-1096. https://doi.org/10.1378/chest.109.4.1093.

11. Chen A, Ho YS, Tu YC, Tang HS, Cheng TC. Diaphragmatic defect as a cause of massive hydrothorax in cirrhosis of liver. J Clin Gastroenterol. 1988;10(6):663-666. https://doi.org/10.1097/00004836-19881200000019.

12. Zenda T, Miyamoto S, Murata S, Mabuchi H. Detection of diaphragmatic defect as the cause of severe hepatic hydrothorax with magnetic resonance imaging. Am J Gastroenterol. 1998;93(11):22882289. https://doi.org/10.1111/j.1572-0241.1998.00639.x.

13. Lazaridis KN, Frank JW, Krowka MJ, Kamath PS. Hepatic hydrothorax: pathogenesis, diagnosis, and management. Am J Med. 1999;107(3):262-267. https://doi.org/10.1016/s0002-9343(99)00217-x.

14. Alagiakrishnan K, Patel PJ. Left-sided hepatic hydrothorax with ascites. Int J Clin Pract. 1999;53(3):225-226. 
15. Rubinstein D, McInnes IE, Dudley FJ. Hepatic hydrothorax in the absence of clinical ascites: diagnosis and management. Gastroenterology. 1985;88(1 Pt 1):188-191. https://doi.org/10.1016/ s0016-5085(85)80154-2.

16. Singer JA, Kaplan MM, Katz RL. Cirrhotic pleural effusion in the absence of ascites. Gastroenterology. 1977;73(3):575-577.

17. Kirsch CM, Chui DW, Yenokida GG, Jensen WA, Bascom PB. Case report: hepatic hydrothorax without ascites. Am J Med Sci. 1991;302(2):103-106. https://doi.org/10.1097/00000441-19910800000007.

18. Singh A, Bajwa A, Shujaat A. Evidence-based review of the management of hepatic hydrothorax. Respiration. 2013;86(2):155-173. https://doi.org/10.1159/000346996.
19. Borchardt J, Smirnov A, Metchnik L, Malnick S. Treating hepatic hydrothorax. BMJ. 2003;326(7392):751-752. https://doi.org/10.1136/ bmj.326.7392.751.

20. Liu LU, Haddadin HA, Bodian CA, Sigal SH, Korman JD, Bodenheimer $\mathrm{HC} \mathrm{Jr}$, et al. Outcome analysis of cirrhotic patients undergoing chest tube placement. Chest. 2004;126(1):142-148. https://doi.org/10.1378/ chest.126.1.142.

21. Orman ES, Lok AS. Outcomes of patients with chest tube insertion for hepatic hydrothorax. Hepatol Int. 2009;3(4):582-586. https://doi. org/10.1007/s12072-009-9136-z.

Conflicto de intereses: Los autores declaran no tener conflicto de intereses. 